\title{
A Study on the Performance Prediction Model for Partial Casing Treatments
}

\author{
Zhenkun Zhu ${ }^{1, a}$, Wei yuan ${ }^{1, b}$, Qiushi $\mathrm{Li}^{1, \mathrm{c}}$ and Zhiping $\mathrm{Li}^{1, \mathrm{~d}}$
}

${ }^{1}$ National Key Laboratory of Science and Technology on Aero-Engines Aero-thermodynamics, School of Energy and Power Engineering, Beihang University, Beijing 100191, China.

azzkun90@163.com, ${ }^{b}$ yuanwei@buaa.edu.cn, ${ }^{\mathrm{a}}$ liqs@buaa.edu.cn, ${ }^{\mathrm{d}}$ leezip@buaa.edu.cn

Keywords: performance prediction; casing treatment; modeling; parallel compressor; expansion of stall margin.

\begin{abstract}
In order to estimate the performance of partial casing treatments, this study improved the classic parallel compressor model, which divides a partial casing treatment compressor into a casing treatment sub-compressor and solid-wall sub-compressor in the circumferential direction. According to the characteristics of a partial casing treatment, the assumptions of the classic parallel compressor model were modified to conduct a performance prediction calculation for a compressor with a partial casing treatment. In this study, a transonic compressor was evaluated and compared using the experiment results. The research results indicated that if the characteristic lines of axial solid-wall and casing treatment compressors were given, this model could well predict the characteristics of the partial casing treatment, no matter what the proportion of the slot area was, and the results were found to be satisfactory.
\end{abstract}

\section{Introduction}

Casing treatment technology is an important means for improving the stall margin of fans/compressors. It was discovered by Koch et al. ${ }^{[1-5]}$ in an experiment. Because of its simple structure and good capacity for improving the stall margin, foreign and Chinese researchers have performed a large number of studies on the technology. The research of Fabri and Reboux ${ }^{[6]}$ showed that casing treatment can improve the stall margin by $20 \%$ to $40 \%$ and that the pressure ratio near the stall point relatively increases. Takata and Tsukuda ${ }^{[7]}$ compared the influence of various treatment slot structures on a compressor's stall margin and found that an axial open/closed skewed slot has the best effect on improving the stall margin, followed by an axial straight slot; conversely, a blade-angle slot and circumferential groove has almost no effect on the stall margin, and an axial reversing skewed slot, which slants in the opposite radial direction, reduces the stall margin. They also discovered that when the slant direction of an axial skewed slot is identical to the rotating direction of the rotor, the compressor has the widest stall margin; when the axial slot does not slant, the stall margin narrows and when it slants against the rotating direction of the rotor, the stall margin is the narrowest. Maozhang Chen of BeihangUniversity improved the design of an engine's casing treatment and significantly widened the stall margin. Later, Jiang et al. conducted further experimental research on the casing treatment of a low-speed compressor ${ }^{[8]}$. XiaoFeng Sun regarded casing treatment as a flexible wall and established a three-dimensional stability model, which took the influence of casing treatment into account ${ }^{[9]}$. Du et al. of ShanghaiJiaotongUniversity and Liu et al. of NorthwesternPolytechnicalUniversity researched the circumferential groove, axial slot, and recess-vaned casing treatment by experiments and analyzed the impact of a casing treatment's structure parameters on the stall margin and efficiency ${ }^{[10,11]}$. Professor YaJun Lu ${ }^{[12]}$ of BeihangUniversity considered that the direction of jet flow from the treatment slot and the flow loss would affect the stall margin of the casing treatment and designed a new type of casing treatment structure with an arc-skewed slot. The experimental results ${ }^{[13]}$ indicate that this kind of treatment slot has a slight influence on compressor efficiency while increasing the stall margin significantly, so it would be a comparatively better type of casing treatment structural form.

Greitzer ${ }^{[14]}$ summarized the various casing-treatment technologies that had been developed and pointed out that all casing treatments increase the stall margin at the cost of lost efficiency and that the 
efficiency loss increases as the stall margin expands. In order to further reduce the losses caused by a casing treatment, research ${ }^{[15]}$ found that asymmetric casing treatment slots (using a partial casing treatment) could improve the stall margin and simultaneously decrease the negative effect of the casing treatment slots on compressor efficiency. Thus, to design a partial casing treatment that meets the required performance, we decided to perform a complete-circle fan/compressor numerical calculation on different partial casing treatment layouts. This will involve a large number of calculations and consume considerable time. Conducting a complete-circle numerical calculation on the partial casing treatment of a multistage fan/compressor can be very difficult, and the computing resources and cost would be unbearable. In order to efficiently and effectively estimate the characteristics of a partial casing treatment, this study presents a performance prediction model for such treatments. The model uses the characteristic line of solid-wall and complete-circle casing treatment to estimate the characteristics of treatments with different circumferential proportions. In this way, we will be able to predict the characteristics of the designed partial casing treatment before the experiments.

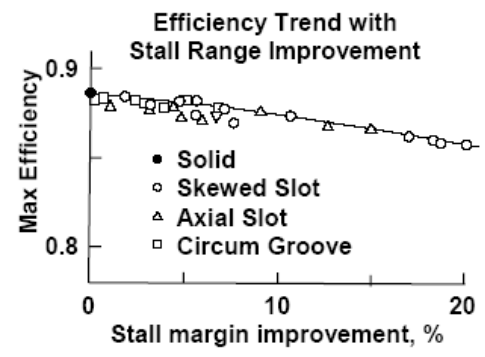

Fig. 1Relationship between compressor peak efficiency and stall margin improvement for various casing treatments ${ }^{[14]}$
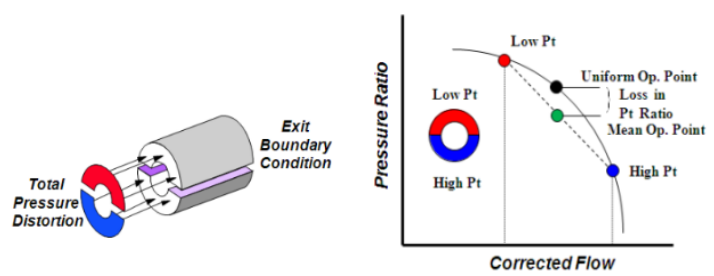

Fig. 2 Characteristic curve of the parallel compressor theory

\section{Performance Prediction Model for Partial Casing Treatments}

The assumptions for a performance prediction model for partial casing treatments were based on the assumptions for the classic parallel compressor model, although some modifications were necessary. Pearson and McKenzie ${ }^{[17]}$ first proposed the parallel compressor model in the late 1950s. Their basic idea was to divide a compressor with inlet circumferential distortion into distortion and non-distortion regions (as depicted in Fig. 2). They presumed that these regions worked independently under their respective inlet conditions, so the inlet distortion compressor was simplified as two independent sub-compressors with uniform inlet flow. The characteristics of the sub-compressors could then be determined, and the characteristics of the distortion compressor could be achieved by a mixing method. The assumptions for the classic parallel compressor model are as follows:

1) The inlet flow conditions of each sub-compressor are different and independent from each other, and the exchange of momentum, mass, or energy is ignored;

2) Each sub-compressor works in parallel and has the same outlet static pressure;

3) Each sub-compressor works under the compressor's characteristic line at uniform inlet flow conditions;

4)Once the mass flow of a sub-compressor reaches the compressor's stall margin boundary at uniform inlet flow conditions, the entire compressor is thought to reach the stall margin boundary.

Considering the basic idea of parallel compressors, we can divide the partial casing treatment into two sub-regions: the casing treatment region and the solid-wall region (as depicted in Fig. 4). Comparing the difference between the partial casing treatment and inlet distortion compressor, we find that the inlet parameters of the partial casing treatment compressor are the same, whereas the environments of its inner flow field are different. Therefore, we can change the first assumption of the parallel compressor in this way: "The inlet flow conditions of each sub-compressor are the same but their inner flow environments are different, and the exchange of momentum, mass, or energy between sub-compressors will be ignored." Because their outlet conditions are the same, the second 
assumption for a parallel compressor (that each sub-compressor has the same outlet static pressure) is also applicable to a partial casing treatment compressor. The third assumption can be altered as follows, in the case of partial casing treatment: "Each sub-compressor works independently under its own characteristic line. This means that the casing treatment sub-compressor works under the characteristic line of the complete-circle casing treatment compressor, and the solid-wall sub-compressor works under the characteristic line of the complete-circle solid-wall compressor." The fourth assumption is the stall margin boundary criterion, according to the experimental findings for a partial casing treatment with different circumferential angles of the casing treatment region ${ }^{[15]}$ : "The mass flow of the stall margin boundary has a linear relationship with the circumferential angle of the casing treatment region (as depicted in Fig. 3)"; thus, the fourth assumption for a partial casing treatment is as follows: "The mass flow of the stall margin boundary is determined by the linear relationship between the mass flow of the stall margin boundary and the circumferential angle of the casing treatment region."

Through the above analysis, the assumptions for the performance prediction model for partial casing treatments can be summarized as follows:

1) The inlet flow conditions of each sub-compressor are the same, but their inner flow environments are different, and the exchange of momentum, mass, or energy between sub-compressors will be ignored;

2) Each sub-compressor works in parallel with the others and has the same outlet static pressure;

3) Each sub-compressor works independently under its own characteristic line;

4) The mass flow of the stall margin boundary is determined by the linear relationship between the mass flow of the stall margin boundary and the circumferential angle of the casing treatment region.

On the basis of the above assumptions, we can obtain the characteristics of a partial casing treatment by calculating the respective sub-compressor parameters and mixing them in a certain manner.

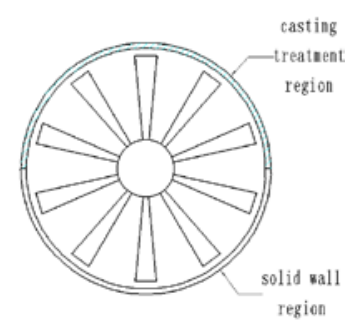

Fig. 3 Partial casing treatment

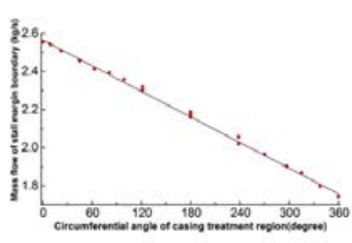

Fig. 4 The relation between the mass flow of the stall margin and the circumferential angle of the casing treatment region

\section{Model Calculations}

All of the experimental data used for calculating the performance prediction model for partial casing treatments were taken from documents ${ }^{[15]}$. These documents illustrate the relevant experiments on partial casing treatments in transonic- and subsonic-speed experimental platforms, respectively. The computational process of the model is described as follows:

1) Calculating the sub-compressor parameters:

According to the second and the third assumptions, we can assume the compressor's outlet static pressure to be " $\mathrm{p}$ ", and because the characteristic line of the sub-compressor is known, we can use the gas dynamic function obtain two points on the characteristic line whose static pressure is "p". Meanwhile, the inlet area of each sub-compressor can be calculated according to the proportions of its circumferential regions. The mass flow, efficiency, and pressure ratio of each sub-compressor can then be determined, and the outlet total temperature and total pressure of each sub-compressor can be obtained by reversing the efficiency and pressure ratio formula.

2) Calculating the compressor parameters after mixing:

According to the laws of mass conservation, momentum conservation, and energy conservation, we can acquire the outlet total temperature and total pressure of the compressor after mixing. We can then obtain the efficiency and pressure ratio of the compressor under this outlet static pressure from 
the efficiency and pressure ratio formula. Afterwards, we can substitute the outlet static pressure for another one and acquire more points on the characteristic line of the compressor by repeating the above process.

3) Estimating the stall point:

We can assume the proportions of the casing treatment and solid-wall regions to be $f_{1}$ and $f_{2}$, respectively, and the mass flow of the stall point of the complete-circle casing treatment sub-compressor and solid-wall sub-compressor to be $m_{n s 1}$ and $m_{n s 2}$, respectively. Then the mass flow of the stall point of the compressor after mixing should be $m=f_{1} m_{n s 1}+f_{2} m_{n s 2}$

\section{Testing of the Partial Casing Treatment Prediction Model}

\subsection{Brief Introduction to the Experimental Tables}

The subsonic-speed experimental platform ${ }^{[16]}$ is a single-stage axial compressor with subsonic speed. It is composed of an inlet guide vane (IGV), rotor row, and stator row. The time average gas dynamic performance parameters of the compressor at the design point are as follows: mass flow $\mathrm{M}=$ $2.9 \mathrm{~kg} / \mathrm{s}$, total pressure rise , and adiabatic efficiency $\eta=86.5 \%$ The other main design parameters are presented in Table 1.

Table 1 Design parameters of a transonic-speed compressor rotor ${ }^{[16]}$

\begin{tabular}{ccccccc}
\hline $\begin{array}{c}\text { Number of } \\
\text { blades }\end{array}$ & $\begin{array}{c}\text { Ratio of } \\
\text { shroud and } \\
\text { hub }\end{array}$ & $\begin{array}{c}\text { Chord } \\
\text { length(mm) }\end{array}$ & $\begin{array}{c}\text { aspect } \\
\text { ratio }\end{array}$ & $\begin{array}{c}\text { shroud } \\
\text { gap(mm) }\end{array}$ & $\begin{array}{c}\text { Design rotating } \\
\text { speed(rpm) }\end{array}$ & $\begin{array}{c}\text { Blade tip } \\
\text { tangential } \\
\text { speed(m/s) }\end{array}$ \\
\hline 17 & 0.565 & $80.5 \mathrm{~mm}$ & 0.956 & $0.9 \mathrm{~mm}$ & $22000 \mathrm{rpm}$ & $409.85 \mathrm{~m} / \mathrm{s}$ \\
\hline
\end{tabular}

\subsection{Model Verification of Transonic-Speed Experimental Platform}
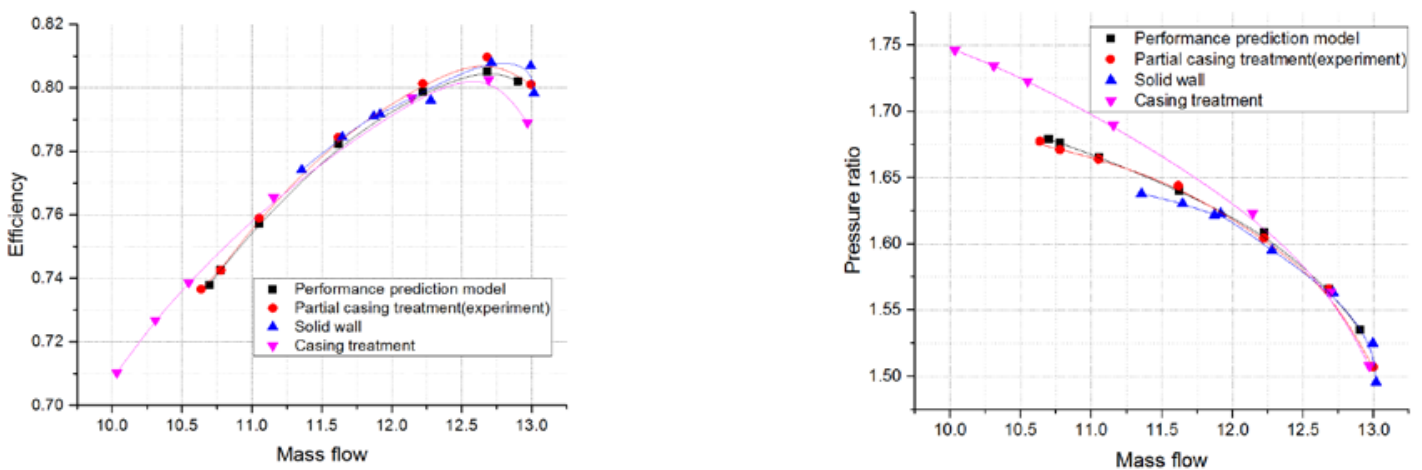

(1) Ninety-eight percent of the designed rotating speed
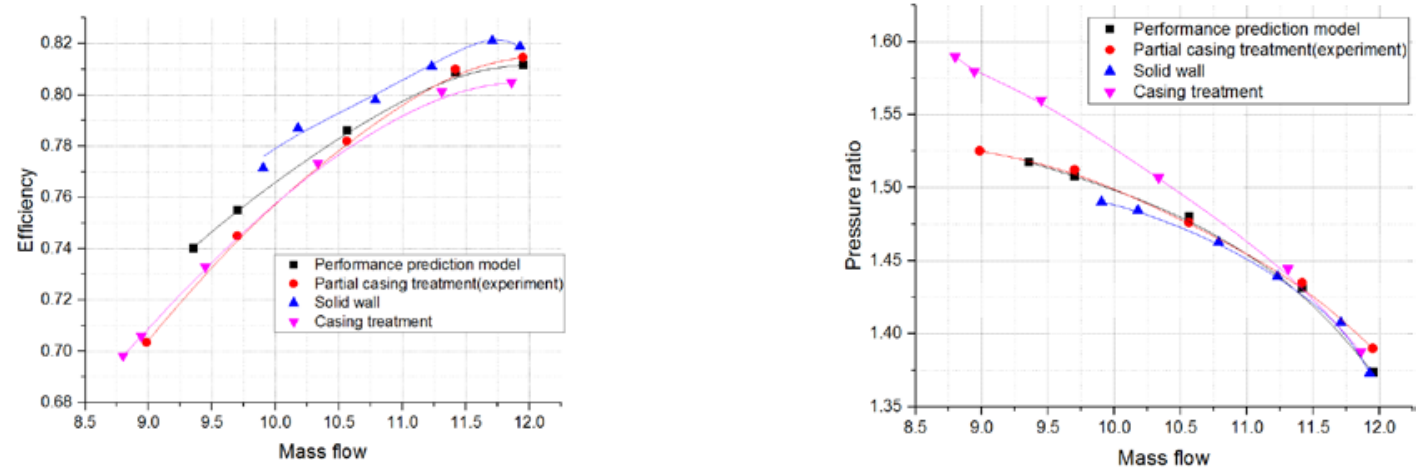

(2) Eighty-eight percent of the designed rotating speed

Fig. 5 Comparison of the experimental and predicted values of a transonic-speed compressor at two rotating speeds

The experimental and prediction characteristics of a transonic-speed compressor at $98 \%$ and $88 \%$ of the designed rotating speed are compared in Fig. 5 (1) and (2), from document ${ }^{[16]}$, respectively. 
According to a qualitative analysis of the characteristic line at these rotating speeds, the trend of the prediction characteristic line of the partial casing treatment is generally the same as the experimental results. However, the deviation between the predicted and experimental efficiency gradually expands as the mass flow decreases when it is at the $88 \%$ rotating speed. However, we can see that the prediction characteristic line is still between the solid-wall characteristic line and casing treatment characteristic line, which agrees with the expected results. Moreover, from the prediction of the peak efficiency point and near-stall margin point, we can see that the peak efficiency can be accurately predicted at the two rotating speeds. The evaluation of the near-stall margin point at the $98 \%$ rotating speed is also reasonably accurate, whereas at the $88 \%$ rotating speed, the prediction mass flow of the stall margin point is slightly smaller than the experimental value, and the stall margin point efficiency is greater than the experimental result. In order to quantitatively analyze the accuracy of the performance prediction model for partial casing treatments, this study started with a view to improving the stall margin of the partial casing treatment and selected several important characteristic parameters to describe its stall margin, including the peak efficiency point parameter, near-stall point parameter, and stall margin to test.

\subsubsection{Peak Efficiency Point Prediction Results}

To verify the accuracy of the prediction model for partial casing treatments, this study first made a comparison of the peak efficiency point parameters of the prediction and experimental characteristics, as depicted in Table 2. It can be seen that at the two rotating speeds, the prediction and experimental mass flows at the peak efficiency point are almost equal. Furthermore, we can see that the prediction and experimental values are nearly at the same level according to the efficiency and pressure ratio parameters. It is thus clear that the prediction model can well predict the experimental value near the peak efficiency point, which also proves the validity of the prediction model at that point.

Table 2 Main characteristic parameters of the prediction and experimental characteristic peak efficiency points

\begin{tabular}{ccccc}
\hline & & Mass flow & Efficiency & $\begin{array}{c}\text { Pressure } \\
\text { ratio }\end{array}$ \\
\hline $\begin{array}{c}\text { 98\% design } \\
\text { rotating } \\
\text { speed }\end{array}$ & Experimental & 12.681 & $80.96 \%$ & 1.5657 \\
\cline { 2 - 5 } & Prediction & 12.680 & $80.52 \%$ & 1.5660 \\
\hline $\begin{array}{c}88 \% \text { design } \\
\text { rotating } \\
\text { speed }\end{array}$ & Experimental & 11.9492 & $81.47 \%$ & 1.3900 \\
\cline { 2 - 5 } & Prediction & 11.9492 & $81.17 \%$ & 1.3737 \\
\hline
\end{tabular}

\subsubsection{Near-Stall Point Prediction Results}

The experimental and prediction mass flows are almost the same when the compressor is near the stall point at the $98 \%$ rotating speed, whereas the prediction mass flow is slightly smaller than the experimental data at the $88 \%$ rotating speed. By observing the prediction value in Table 3 and Fig. 5 (2), we can see that the prediction mass flow is slightly larger than the experimental value when the compressor is near the stall point at the $88 \%$ rotating speed, Also, the prediction efficiency is $3.69 \%$ higher than the experimental value, whereas the prediction pressure ratio is somewhat closer to the experimental value. It can be seen from the characteristics of Fig. 5 (2) that the prediction stall point is between the characteristic lines of the solid wall and the casing treatment. This conforms to the theoretical value for partial casing treatment performance; hence, there are two main reasons for error in the prediction value at medium rotating speeds: first, the assumptions for the prediction model neglected the exchange of mass, momentum, and energy between the sub-compressors, but these exchanges can be considerable when the compressor is near the stall point at medium rotating speeds, and this could influence the precision of the prediction model to a certain degree. Second, the experimental conditions will cause additional errors. By examining Table3, we can also see that the prediction accuracy is higher when the compressor is at a high rotating speed. 
Table 3 Main characteristic parameters of the prediction and experimental characteristics near the stall point

\begin{tabular}{clccc}
\hline & & Mass flow & Efficiency & Pressure ratio \\
\hline $\begin{array}{c}\text { 98\% design } \\
\text { rotating } \\
\text { speed }\end{array}$ & Experimental & 10.64 & $73.66 \%$ & 1.678 \\
\cline { 2 - 5 } $\begin{array}{c}88 \% \text { design } \\
\text { rotating } \\
\text { speed }\end{array}$ & Experimental & 10.69 & $73.79 \%$ & 1.679 \\
\cline { 2 - 5 } & Prediction & 9.35 & $70.35 \%$ & 1.525 \\
\hline
\end{tabular}

\subsubsection{Near-Stall Point Prediction Results}

SurveyStall margin is a parameter used to measure the stable working range of a compressor. Its definition formula is as follows:

$$
\phi=\left(\frac{\pi_{n s}^{*} / \dot{m}_{n s}}{\pi_{p e}^{*} / \dot{m}_{p e}}-1\right) \times 100 \%
$$

where, $\pi_{n s}^{*}$ is the pressure ratio near the stall point, $\dot{m}_{n s}$ is the mass flow near the stall point, $\pi_{p e}^{*}$ is the pressure ratio at the peak efficiency point, and $\dot{m}_{p e}$ is the mass flow at the peak efficiency point. A stall margin that is defined this way is called a comprehensive margin. In order to sufficiently measure the precision of the prediction model, we can define the mass flow and pressure ratio margins to observe the accuracy of the prediction characteristics. The definition method for the mass flow and pressure ratio margins is similar to that for the comprehensive margin. The definition formulas are as follows:

$$
\begin{aligned}
& \phi_{m}=\frac{\dot{m}_{p e}}{\dot{m}_{n s}}-1 \times 100 \% \\
& \phi_{\mathrm{p}}=\left(\frac{\pi_{n s}}{\pi_{p e}}-1\right) \times 100 \%
\end{aligned}
$$

Table 5 lists the experimental and prediction characteristic values for the three kinds of margins at the $98 \%$ and $88 \%$ rotating speeds. It can be seen that when the compressor is at the $98 \%$ rotating speed, the prediction and experimental values of the mass flow margin, comprehensive margin, and pressure ratio margin are close, with the greatest difference value being no more than $0.59 \%$. It is also seen that when the compressor is at the $88 \%$ rotating speed, the prediction values of the mass flow margin and comprehensive margin are slightly smaller than the experimental values, but the prediction value of the pressure ratio margin is slightly larger than the experimental value. Because the definition formula for the comprehensive margin includes the mass flow and pressure ratio, a change in these parameters will also reveal a change in the comprehensive margin. From the comparison in Fig. 5 (2), we can see that when the compressor is at the $88 \%$ rotating speed, the prediction efficiency of the near-stall margin point is higher than expected, but the prediction mass flow is smaller. This is why the mass flow and pressure ratio margins show a comparatively large deviation when the compressor is at the $88 \%$ rotating speed.

Table 5 Prediction margin and experimental margin

\begin{tabular}{lcccc}
\hline & & Mass flow margin & Comprehensive margin & Pressure ratio margin \\
\hline $\begin{array}{l}\text { 98\% design } \\
\text { rotating }\end{array}$ & Experimental & $19.18 \%$ & $27.76 \%$ & $7.17 \%$ \\
\cline { 2 - 5 } speed & Prediction & $18.62 \%$ & $27.17 \%$ & $7.22 \%$ \\
\hline $\begin{array}{l}88 \% \text { design } \\
\text { rotating }\end{array}$ & Experimental & $33.06 \%$ & $45.97 \%$ & $9.71 \%$ \\
\cline { 2 - 5 } speed & Prediction & $27.80 \%$ & $41.18 \%$ & $10.50 \%$ \\
\hline
\end{tabular}

From the above comparisons, we can see that if the proportion of the casing treatment region is the same, the prediction parameters of the peak efficiency point and near-stall point, as well as the margins, are all consistent with the experimental values, particularly when the compressor is rotating at high speed. Moreover, when it is rotating at medium speed, the prediction parameters of the peak efficiency point are found to be satisfactory, and the prediction mass flow and pressure ratio of the 
near-stall point show only a slight deviation compared with the experimental values. Although the prediction and experimental efficiencies are somewhat different, the prediction results are theoretically acceptable. Furthermore, the above results also prove that the performance prediction model for partial casing treatments is accurate under transonic-speed conditions, which indicates that the model can be applied to predict the characteristics of the partial casing treatment of a transonic compressor.

\section{Conclusion}

1) This study puts forward a new kind of performance prediction model for partial casing treatments. It can quickly predict the performance of a partial casing treatment when the characteristics of the solid wall and the casing treatment are given;

2 ) The model can provide relatively precise prediction results for transonic-speed compressors as well as subsonic-speed compressors;

3) The precision of the model is related to the actual situation, which means that when the exchange of momentum, mass, and energy between the sub-compressors is limited, the prediction accuracy is higher. Otherwise, the prediction precision is diminished.

\section{Acknowledgement}

This investigation was supported by the National Natural Science Foundation of China (51176005).

\section{References}

[1] Koch, C. C., and Smith, L. H., Jr. "Experimental Evaluation of Outer Case Blowing or Bleeding of a Single Stage Axial Flow Compressor, Part II-Performance of Plain Casing Insert Configuration with Undistorted Inlet Flow and Boundary Layer Trip[R],” NASA CR-54588, 1968

[2] Koch, C. C., and Smith, L. H., Jr. "Experimental Evaluation of Outer Case Blowing or Bleeding of a Single Stage Axial Flow Compressor, Part III-Performance of Blowing Insert Configuration No.1[R],” NASA CR-54589, 1968

[3] Koch, C. C., and Smith, L. H., Jr. "Experimental Evaluation of Outer Case Blowing or Bleeding of a Single Stage Axial Flow Compressor, Part IV_-Performance of Plain Bleeding Insert Configuration No.3[R],” NASA CR-54590, 1968

[4] Koch, C. C., and Smith, L. H., Jr. "Experimental Evaluation of Outer Case Blowing or Bleeding of a Single Stage Axial Flow Compressor, Part V-Performance of Plain Casing Insert Configuration with Distorted Inlet Flow[R],” NASA CR-54591, 1969

[5] Koch, C. C., and Smith, L. H., Jr. "Experimental Evaluation of Outer Case Blowing or Bleeding of a Single Stage Axial Flow Compressor, Part VI—Final Report[R].” NASA CR-54592, 1970

[6] Fabri J., and Reboux J., "Effect of Outer Casing Treatment and Tip Clearance on Stall Margin of a Supersonic Rotating Cascade,” ASME Paper 75-GT-95

[7] Takata H., and Tsukuda Y., "Stall Margin Improvement by Casing Treatment—Its Mechanism and Effectiveness,”ASME Journal of Engineering for Power, Vol.99, 1977

[8] Zhang H.,Jiang H., and Liu H., "The tip flow at the exit of rotor with a fan-shape treated casting”Journal of Engineering Thermophysics, Vol.23,2002

[9] Sun X. F., "On the Relation Between the Inception of Rotating Stall and Casing Treatment," AIAA Paper 96-2579

[10] Liu Z., “A review of studies on casing treatment of axial-flow compressor,"Northwestern Polytechnical University,PA01647 
[11] Du H., Zhu J., Chu W., Liu Z., “Optimization of Structural Parameters of Recess Vanned Casing Treatment,” Journal of Propulsion Technology,Vol.19, No.1, 1998

[12] Li L., Yu Q., Lu Y.,"Experimental research of casting treatment on single stage transonic fan [J],’Journal of Propulsion Technology, Vol. 21, No.3, 2000

[13] Yuan W.,"The Research on The Influence of Casing Treatment Geometry on Compressor Characteristic [D],’Beihang University, Beijing, 2003

[14] Greitzer, E. M., Nikkanen, J. P., Haddad, D. E., et.al.,“A Fundamental Criterion for the Application of Rotor Casing Treatment[J],”Journal of Fluids Engineering, Vol. 101, No.4, 1979, pp. 237-243

[15] Liu J., "Exploration on non-axisymmetric cooperating control technology for unsteady vortex flow in compressors [D],”Beihang University,Beijing, 2009

[16] Li M.,"Mechanism Investigation about Inlet Flow Distortion and its extending stability by Casing Treatment on a Transonic compressor [D],’Beihang University, Beijing, 2014

[17] Pearson H., and McKenzie A. B.,"Wakes in Axial Compressors,”Journal of the Royal Aeronautical Society, 1959 